\title{
AKUPUNKTURKONGRESS IN TIMMENDORF ZIEHT JUNGE KOLLEGEN AN
}

\section{Liebe Kolleginnen und Kollegen,}

auch dieses Jahr neigt sich schon wieder dem Ende entgegen. Ich hoffe, Sie können auf ein gutes Jahr zurückblicken. In Bezug auf unseren Verein hatten wir einige Höhepunkte. So waren die Ausbildungs- und Fortbildungskurse wieder sehr gut besucht und die Rückmeldungen durchgängig positiv. Auch die neuen Fortbildungskurse „Master Tung“ und der „Arzthelferinnenkurs“ erhielten gute Kritiken. Der Kongress am Timmendorfer Strand hat allen Beteiligten wieder kollegialen Austausch, Wissensvertiefung und neue Denkanstöße für den Praxisalltag gegeben. Die Wandlungsphase Erde stand im Mittelpunkt der Woche und die verschiedenen Fachvorträge und Workshops zu den Bereichen Akupunktur, Laseranwendung, Darmflora, Blüten- und Kräutertherapie, Implantatnadel und Materialtestung waren gut besucht. Das Konzept der verschiedenen Wandlungsphasen als Aufhänger für die Vorträge hat sich auch dieses Jahr bewährt und wird auch in den nächsten Jahren fortgesetzt. Timmendorf 2017 wird unter dem Wasser-Element stehen und die ersten Vortragsmeldungen versprechen wieder ein buntes und reichhaltiges Programm. Die Aktion Ü50 trifft U40 hat einige jüngere Kollegen motiviert, den Sprung zum Kongress nach Timmendorf zu wagen, sodass wir auch 2017 diese Aktion fortführen wollen.

Der DAA-Berufsverband informiert:

In diesem Jahr kam es zu einer Abmahnwelle, die in Bezug auf unsere Akademie zwei Kollegen betraf. Auch andere Gesellschaften hatten in diesem Umfang Abmahnungen bei ihren Mitgliedern gemeldet bekommen. Aus diesem
Grunde möchten wir darauf hinweisen, dass Sie insbesondere bei der Online-Werbung Akupunktur in Zusammenhang mit der Nennung von Indikationen vermeiden sollten. Sowohl das Zitieren derWHO als auch dieVerwendung der Wortgruppe „kann helfen“ sind kein ausreichender Schutz. Verwenden Sie auch die Texte aus unseren Informationsbroschüren/-plakaten nicht in der OnlineWerbung. Sie sind als Praxisinformation und persönliche Handreichung für den Patienten gedacht, damit dieser eine Entscheidungsgrundlage für die Zustimmung zur Behandlung hat. Die DAA-Mitglieder-Homepage, die wir Ihnen bereitstellen, erfüllt die oben aufgeführten Bedingungen. Die dort abgelegten Informationen sind seriös und defensiv gehalten. Bisher hat es diesbezüglich keine Abmahnungen gegeben. Wir stehen mit anderen Fachgesellschaften und Berufsverbänden in regem Austausch zu diesem Thema. Im neu von der DAA e.V. angebotenen „Basiskurs für Arzthelferinnen " wird der Bereich Werbung und Abmahnung explizit behandelt, sodass Ihre Medizinische Fachangestellte auch für dieses Thema sensibilisiert ist.

Ein frohes und gesegnetes Weihnachtsfest mit den besten Wünschen für 2017

Ihr

B. Ramme

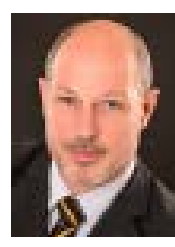

Dr. med. Bernd Ramme

1. Vorsitzender der DAA e.V., Osserstraße 40, D-81679 München Tel. $+4989 / 8145252$

E-Mail des allg.Büros: kontakt@akupunktur.de, Internet:www.akupunktur.de 